\title{
Special section on intelligent \& fuzzy theory in engineering and science
}

\author{
Teresa Guarda ${ }^{\mathrm{a}}$, Isabel Lopes ${ }^{\mathrm{b}}$ and Álvaro Rocha ${ }^{\mathrm{c}, *}$ \\ ${ }^{a}$ State University of Península de Santa Elena, La Libertad, Ecuador \\ ${ }^{\mathrm{b}}$ Polytechnic Institute of Bragança, Bragança, Portugal \\ ${ }^{\mathrm{c}}$ University of Coimbra, Coimbra, Portugal
}

In recent decades, in order to reflect the complex needs of the information society, the field of "Intelligence \& Fuzzy Theory" has continuously expanded its themes and boundaries. As a result, many academic studies have attempted to address complex information science and technology issues from a broader perspective.

Therefore, it is our pleasure to release this issue of Journal of Intelligent \& Fuzzy Systems, which represents cross-cutting research in the field of information technology and artificial intelligence, involving social sciences and engineering technology. The key areas of the selected articles include network algorithms, Internet of Things, and intelligent algorithms.

There are papers focused on network algorithms. In order to optimize the sharing of resources, build a secure and reliable network environment, and meet the increasing security and reliability requirements of new network environments, an article proposes a clustering algorithm that effectively evaluates the resource sharing operation mechanism, at the same time, a resource sharing system based on network algorithm is designed, and the wireless network security standards and protocols are updated and strengthened. A shared model is proposed and built, and an information resource sharing service system

\footnotetext{
*Corresponding author. Álvaro Rocha, University of Coimbra, Coimbra, Portugal. E-mail: amrrocha@gmail.com.
}

is designed. Experimental results show that the algorithm is very effective. It provides effective technical support and theoretical reference for realizing information resource sharing service and constructing a secure network environment.

Meanwhile, there are papers focused on Internet of Things. In order to improve the innovation and development of business and teaching management, a multi-objective genetic algorithm is constructed to introduce Internet of Things technology into enterprise management innovation. Based on the IoT technology, a speech recognition algorithm is proposed based on the network teaching system, and develops the function of the network teaching system. Moreover, the current mature technical framework is compared, the key technologies of the IoT that using the system architecture is determined. The experimental results show that this method has enlightening effect on the management innovation of enterprises under the information environment, and can provide reference for the future integration of teaching and computer technology.

In addition, there are articles focused on intelligent algorithms. In order to improve people's life indicators, an article uses intelligent algorithm to study electronic technology, regional environment, quality of life and other aspects. According to the intelligent algorithm, an article proposes a personalized recommendation model based on multidimensional space, which has some enlightenment 
for the extension of the personalized recommendation algorithm and the improvement of the user management of e-commerce. Moreover, some articles put forward a virtual environment based on virtual reality technology and intelligent algorithm, and analyze the landscape design of some areas. And, an intelligent management scheme is introduced, and a virtual environment based on virtual reality technology and intelligent management scheme is proposed. The experimental results show that the algorithm is effective and the application of intelligent algorithm in environmental design is feasible, and some results have been obtained.
This issue is the continuation of our publishing activities. All the included articles were anonymously reviewed by experts to maintain academic excellence and integrity. We wish to thank all, including also authors and others, who directly and indirectly have been contributing to the release of this issue by their engagement.
Teresa Guarda Isabel Lopes Álvaro Rocha 